\title{
Design and Realization of a Wide-Band EBG Antenna Based on FSS and Operating in the Ku-Band
}

\author{
Lina Moustafa and Bernard Jecko \\ OSA Department, XLim Laboratory, UMR CNRS 6172, University of Limoges, 123 Avenue Albert Thomas, \\ 87060 Limoges Cedex, France \\ Correspondence should be addressed to Lina Moustafa, moustafa.lina@gmail.com
}

Received 14 January 2010; Accepted 25 March 2010

Academic Editor: Wei Hong

Copyright ( $\odot 2010$ L. Moustafa and B. Jecko. This is an open access article distributed under the Creative Commons Attribution License, which permits unrestricted use, distribution, and reproduction in any medium, provided the original work is properly cited.

\begin{abstract}
EBG antennas are used for directive, omnidirectional, and sectoral applications. Nevertheless, the domain of use of these antennas can be extended to other applications where we can take advantage of their interesting performances notably in terms of directivity and compactness. One of these applications can be the broadband application where the needed bandwidths are much larger than the bandwidths offered by actual EBG antenna designs. Many solutions have been proposed in the literature to widen this bandwidth. This paper deals with the EBG band-widening method based on the use of FSS and focuses on the ability of the EBG resonator to have a broadband behavior if its upper interface is built of FSS chosen and arranged adequately. First, the behavior of the new EBG material formed by combining different FSSs, and the one of the new EBG resonator is briefly described. Then, a prototype operating in the Ku-band is proposed to validate the theoretical concept. Finally, an experimental validation is realized.
\end{abstract}

\section{Introduction}

In recent years, a new kind of highly directive and compact antennas has been realized using Electromagnetic Band Gap EBG materials [1-5]. In such periodic structures, the wave propagation is forbidden for given frequency bands and incidence angles [6]. The insertion of a periodicity defect inside the EBG structure enables the creation of an allowed band within the prohibited frequency band. It has been shown that by exciting such a structure within its allowed band, directive antennas can be obtained.

EBG antennas are able to produce gain values higher than $30 \mathrm{~dB}$, thus providing a quasiplanar alternative to the aperture antennas $[7,8]$. These gains can be achieved in presence of a simple feeding source; the antenna may then overcome the complexity of the feeding mechanism used to feed an array of patches. However, these antennas present an extremely narrow radiating bandwidth for high directivity values. Therefore, a compromise is to be considered between, on the one hand, the number of patches required to perform the same directivity as an EBG antenna fed by a single source, and, on the other hand, the desired radiation bandwidth.

More recently, new surfaces have been proposed to replace the EBG antenna upper interface usually conceived of periodic dielectric layers. These surfaces are known as Frequency Selective Surfaces (FSSs) and they have been used to design different types of EBG antennas with directive [9, $10]$, sectoral $[11,12]$, and omnidirectional $[13,14]$ radiation patterns. The use of the FSS in the EBG antenna has enabled the conception of many configurations like the dual band and the low profile designs. Moreover, these surfaces combined together allow, with respect to certain conditions [15], the enhancement of the antenna radiation bandwidth.

This last configuration with combined FSS will be used in this paper, to design a wide-band EBG antenna operating in the Ku-band. The creation method of a double-layer material which provides a broadband performance as well as the EBG resonator behaviour in presence of this material are first reminded. The material is then designed to be later applied into the design of a monosource EBG antenna operating 
around $12 \mathrm{GHz}$. An experimental validation of the wide band concept is finally given.

\section{EBG Broadband Principle Using Double FSS Layers}

One characteristic of the EBG material is to forbid the propagation of electromagnetic waves whose frequencies belong to the material band gap.

The second characteristic is to allow electromagnetic modes to exist within the forbidden frequency band, by introducing defects in the periodic structure. This defect is generally a break of periodicity realized in the middle of the EBG material. The presence of the defect generates an allowed band which is usually represented by a transmission peak inside the material band gap.

Once used to form EBG resonator antennas, the width of this peak constitutes an essential element to determine the antenna radiation bandwidth. The peak width can be modulated by the choice of the defect; nevertheless the allowed bandwidth is mostly narrow, thus limiting the EBG antenna bandwidth. It has been demonstrated in $[15,16]$ that the allowed bandwidth can be increased by the use of a new kind of EBG material combining double FSS layers (Figure 1). The structure used to illustrate this principle operates in the C-band. It is formed by a pass band FSS in the high layer and of a stop-band frequency selective surface in the low layer. The high layer was composed of a metallic grid garnished with ring slots. The low layer was composed of ring slots periodic in $x$-and $y$-directions.

The combination of double layers of frequency selective surfaces in the upper interface of the EBG antenna, with respect to some criteria such as the FSS nature and the distance between FSS $[15,16]$, has been shown advantageous in terms of bandwidth. In particularly, the choice of a lowlayer FSS more reflective than the FSS present in the high layer can participate to the formation of a double-layer material with positive slope reflection phase. The increased phase profile realized in a local frequency band will allow satisfaction of the resonance condition of the EBG cavity in a wide frequency band, consequently increasing the antenna radiation bandwidth.

2.1. New EBG Resonator Dispersion Diagram. The EBG resonator is a waveguide which has the particularity to associate to the wave guidance, the losses caused by the wave leakage through the partially reflecting upper interface. This leakage is responsible of the antenna radiation.

The conditions on the upper interface and on the lower interface which is in general a perfect electric conductor plane fix the values of the normal component $k_{c}$ of the wave vector $k_{0}$ in the cavity

$$
\varphi_{\text {sup }}+\pi-2 k_{c} h=2 n \pi
$$

where $\varphi_{\text {sup }}$ is the reflection coefficient phase of the upper interface, $h$ is the resonator height, and $n$ is integer.

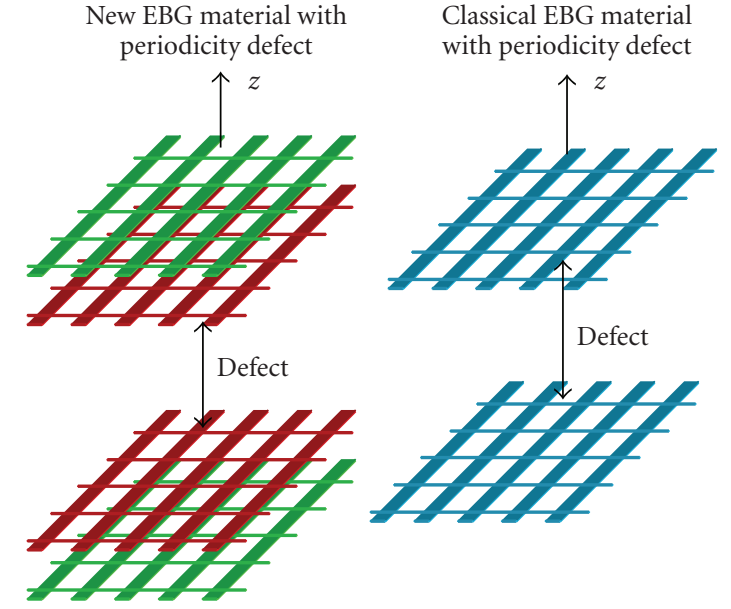

(a)

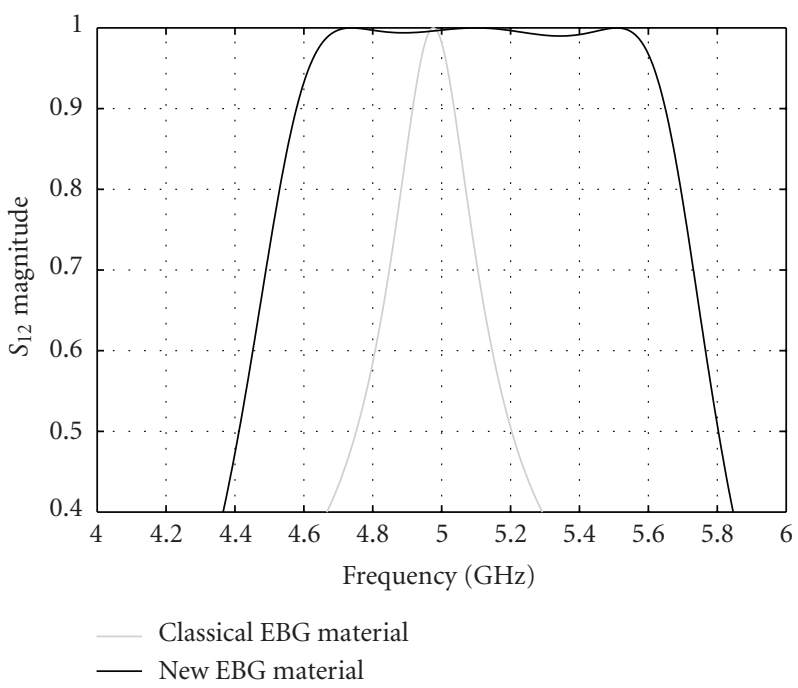

(b)

FIGURE 1: Transmission response shape obtained with the new EBG material with periodicity defect compared to that of a classical EBG material with periodicity defect.

The propagation constant $\Gamma$ of the mode within the guide, which takes into account the losses due to the radiation [17], for a given polarisation

$$
\Gamma(\theta, f)=\sqrt{\left(\frac{(2 n+1) \pi+\ln R_{\text {sup }}+j \varphi_{\text {sup }}}{2 h}\right)-k_{0}^{2}}
$$

$\theta$ is the angle between the normal component $k_{c}$ and the wave vector $k_{0}$ of the mode in the cavity; $f$ is the frequency and $R_{\text {sup }}$ is the reflection coefficient magnitude of the cavity upper interface.

$\Gamma$ is plotted (Figure 2) in the case of the new EBG material. The real part of the propagation constant $\Gamma$, performing the attenuation coefficient $\alpha$ of the cavity mode, is represented in grey. The imaginary part, performing the propagation coefficient $\beta$, is represented in black. The cutoff frequency $f_{c}$ of the waveguide is located at the intersection of both curves. It represents the limit between the evanescent 


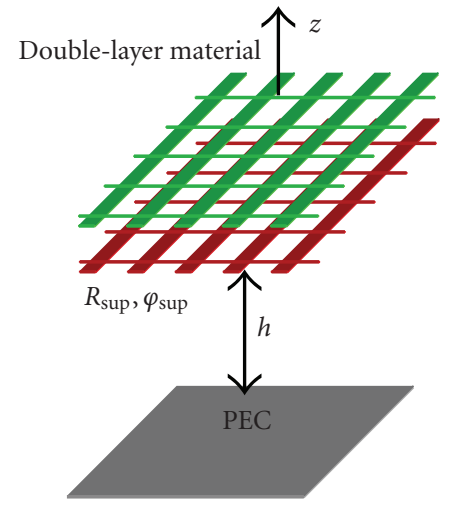

(a)

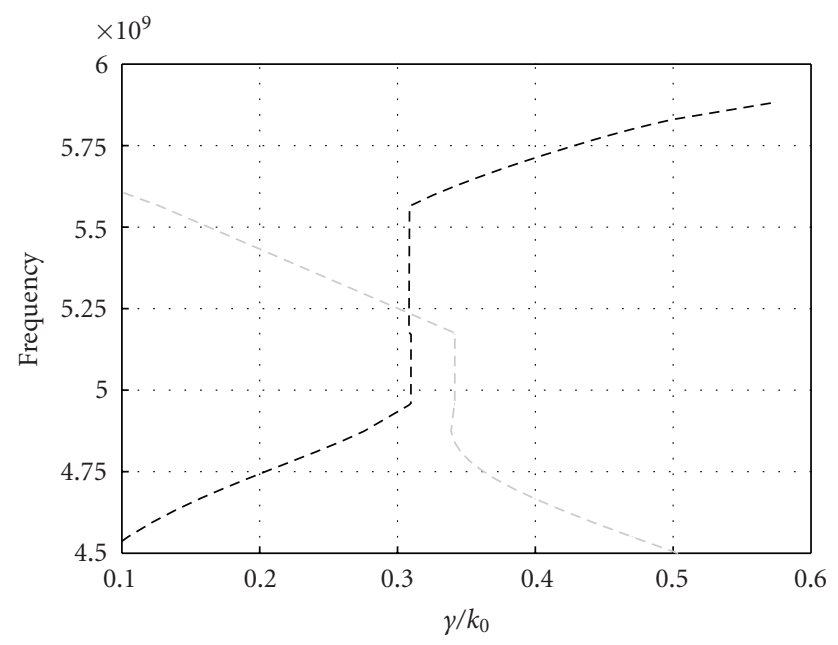

(b)

Figure 2: Dispersion curves obtained by the use of the resonance condition and taking into account the radiation losses.

region $(\alpha>\beta)$ responsible of directive radiations and the propagation region $(\beta<\alpha)$ which causes the appearance of multiple lobes.

For a waveguide with radiation losses, $f_{c}$ corresponds to the frequency for which the real part of $\Gamma$ is equal to its imaginary part $(\alpha=\beta \neq 0)$. Here it is equal to $5.25 \mathrm{GHz}$. Before this frequency, the attenuation coefficient is higher than the propagation coefficient; the mode is almost evanescent and the radiation is normal to the ground plane of the antenna. Contrarily to a classical EBG resonator, there is a range of frequencies for which $\alpha$ and $\beta$ are constant, it is equal to the interval [ $4.95 \mathrm{GHz} ; 5.2 \mathrm{GHz}$ ]: the zone where the mode is evanescent $(\alpha>\beta)$ and hence where the radiation pattern is directive is extended.

After the cutoff frequency, the real part of $\Gamma$ decreases when its imaginary part is maintained constant in a definite frequency range $\left[f_{c}: 5.55 \mathrm{GHz}\right]$. The stagnancy of $\beta$ in a wide frequency band $[4.95 \mathrm{GHz} ; 5.55 \mathrm{GHz}$ ] allows maintaining directive radiation in a wider frequency band. The narrow radiation bandwidth of classical EBG resonators is due to the fast variation of $\beta$ with the frequency.

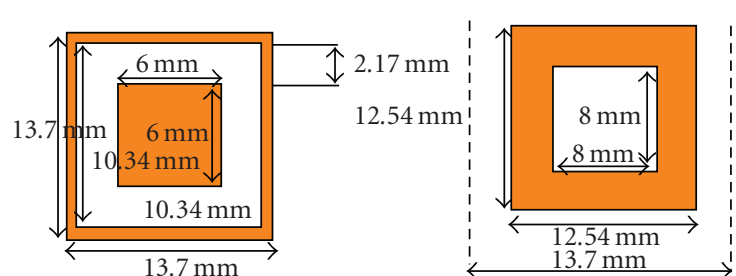

FIGURE 3: Geometry of the FSS layers motifs. On the left, the high layer unit cell. On the right, the low layer unit cell.

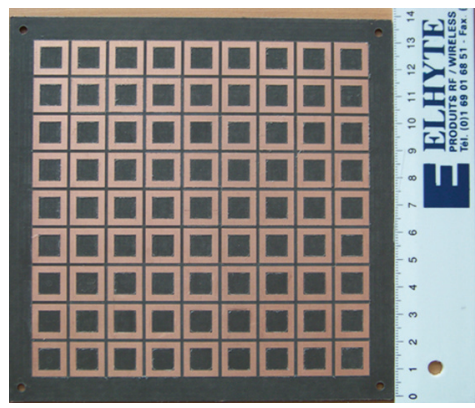

(a)

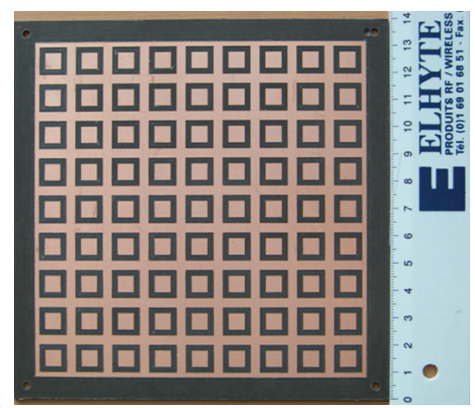

(b)

FIgure 4: (a) The low stage of the double-layer material. (b) The higher stage FSS.

\section{Design of an EBG Antenna Working in the Ku-Band}

3.1. Design of the Partially Reflecting Interface. The principle reminded above will be applied to realize a prototype operating in the Ku-band. Two layers of frequency selective surfaces are used; the low one is a stop-band FSS composed of ring slot unit cells periodic along $x$ - and $y$-directions. The high layer is a pass band FSS built of a metallic grid filled up with square patches. The dimensions of each FSS element are mentioned (Figure 3).

To minimize the dielectric losses and subsequently maximize the frequency bandwidth for which the reflection phase of the double-layer FSS can be inverted, a dielectric substrate with low permittivity and thickness is chosen, that is, RT duroid $5870\left(\varepsilon_{r}=2.33, \operatorname{tg} \delta=0.0012\right.$, and thickness $=1.575 \mathrm{~mm}$ ). Figure 4 shows the layout of the FSS layers printed on one side and on the other side of the substrate board. 


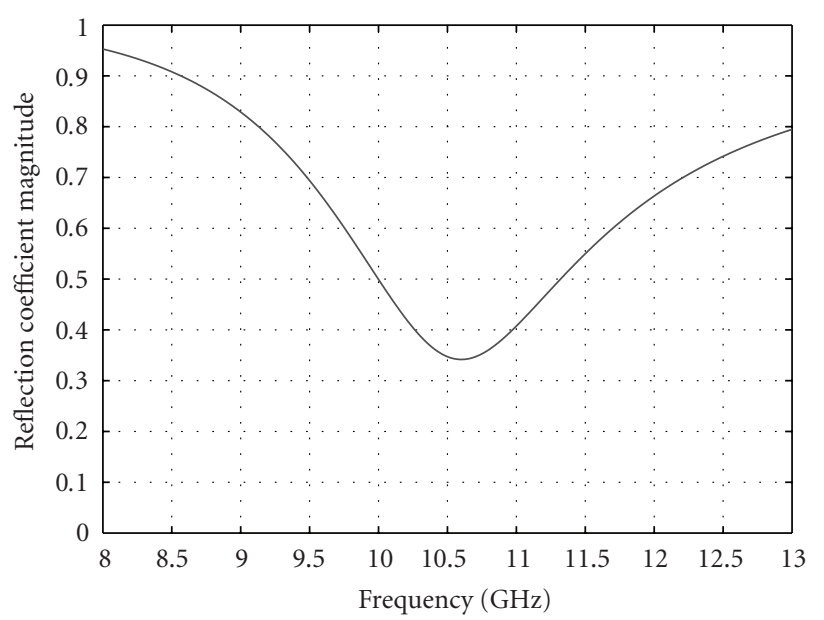

(a)

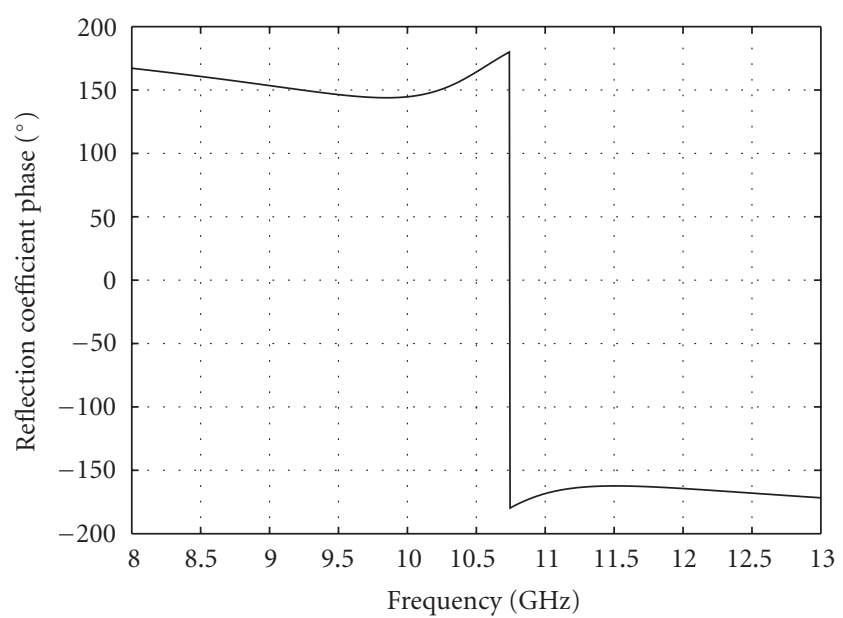

(b)

FIGURE 5: Reflectivity of the double-layer FSS.

The reflectivity of the double-layer material printed on the duroid substrate is pointed out (Figure 5). Unfortunately, even though the substrate has been adequately selected (low permittivity and thickness to maximize the reflectivity magnitude), the reflection coefficient magnitude offered by the structure is still low. A foam substrate has been studied in previous works $[15,16]$, it allows better reflectivity performances (higher reflection magnitude and larger phase inversion have been obtained), this substrate could not have been provided for experimental validation.

The dimensions of the FSS unit cells are chosen in a manner that the total unit cell resonates at $10.5 \mathrm{GHz}$ in proximity to the operating frequency assessed to $12.75 \mathrm{GHz}$ and not exactly at $12.75 \mathrm{GHz}$. So we can take advantage of a higher reflection coefficient magnitude at the operating frequency and in consequence obtain a better directivity in the antenna. Unlikely when the operating frequency is not the same as the double-layer material resonance frequency, the local inversion of the phase slope is not used in an optimal

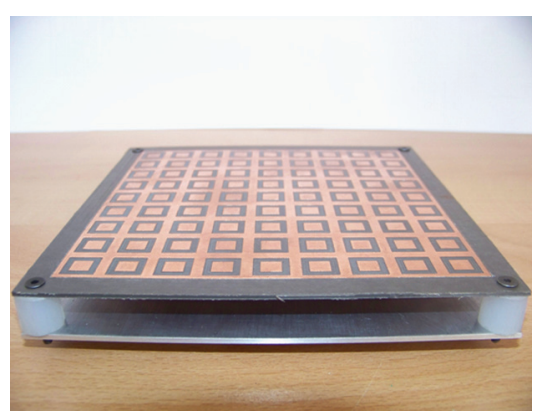

(a)

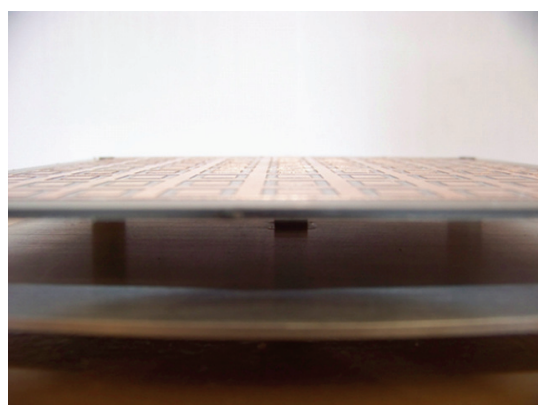

(b)

FIGURE 6: Photography of the complete antenna structure.

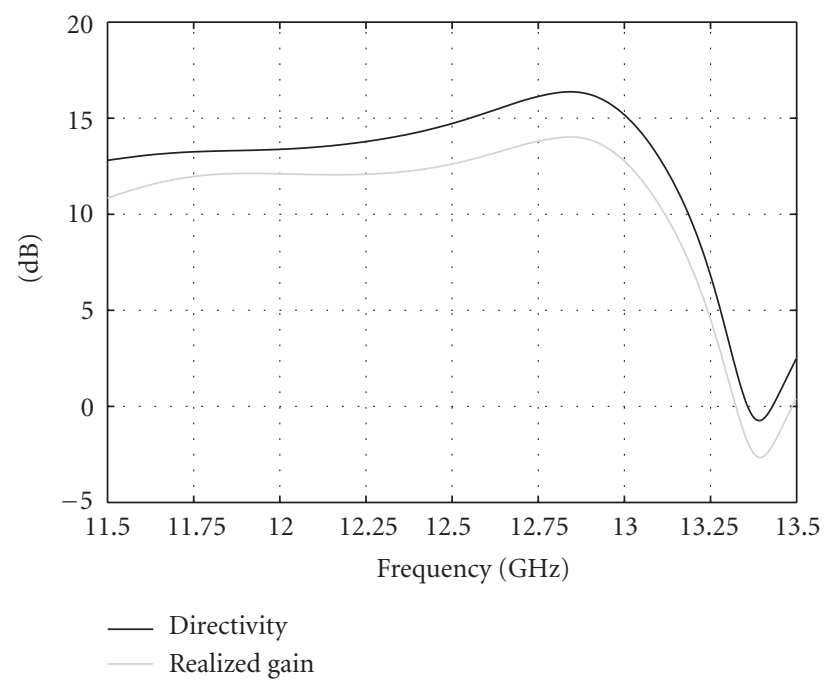

FIGURE 7: Simulated directivity and realized gain of the designed antenna.

way. A compromise between desired maximal directivity and radiation bandwidth has been considered.

3.2. Design of the EBG Antenna. The antenna using the double-layer material studied above is designed (Figure 6). Combined FSSs printed on a duroid board lay in the cavity upper interface and a perfect electric conductor is deposited in the lower interface. Upper and lower interfaces are separated by a cavity height of $12 \mathrm{~mm}$ in order to work at 12.75 GHz. The cavity is fed by a patch-feeding source placed inside the resonator in the proximity to its ground plane. 


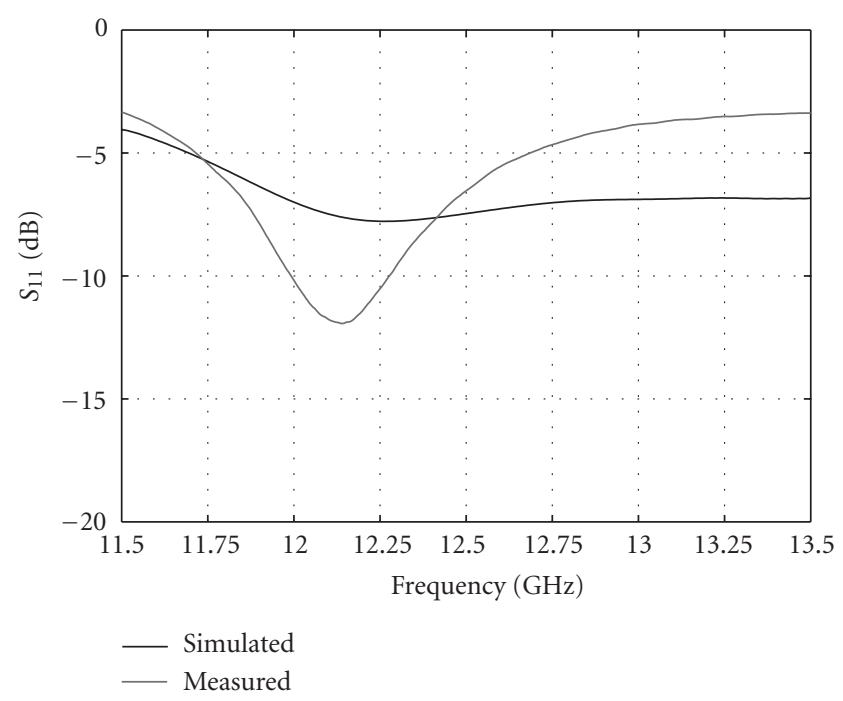

FIGURE 8: Simulated and measured matching of the whole antenna.

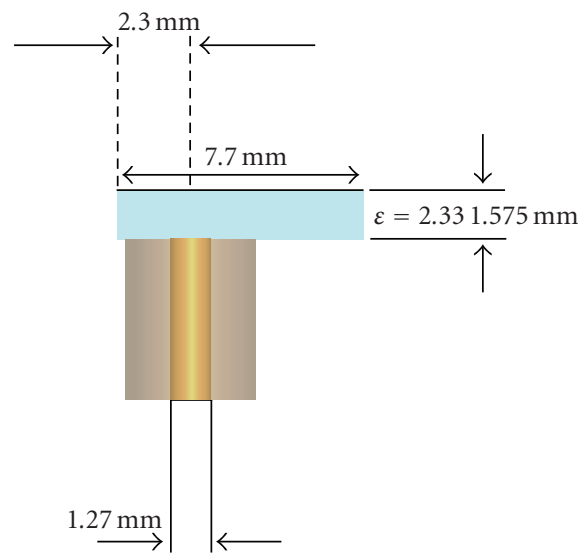

FIGURE 9: The patch source used to excite the EBG resonator.

The patch is printed on the same substrate as the frequency selective surfaces.

The whole antenna structure is studied and its performances are evaluated. Figure 7 represents the simulated directivity and realized gain of the antenna. The maximal directivity has a value of $16 \mathrm{~dB}$ and it is obtained at about $12.75 \mathrm{GHz}$ as expected by the cavity height value. The radiation bandwidth, at $-3 \mathrm{~dB}$ of maximal directivity, is equal to $14 \%$, which is equivalent to an improvement of at least 3\% compared to classical EBG antennas (a classical EBG antenna has a radiation bandwidth of at best $11 \%$ for the same value of directivity [18]). The realized gain curve has a maximal value at the same frequency as the directivity curve and it has the same radiation bandwidth. The privilege of such a structure lies on the maintain of the radiation bandwidth corresponding to the realized gain in regard to the radiation bandwidth corresponding to the directivity. A decline of about $2 \mathrm{~dB}$ is observed throughout the radiation bandwidth. This is due to the high $S_{11}$ magnitude value

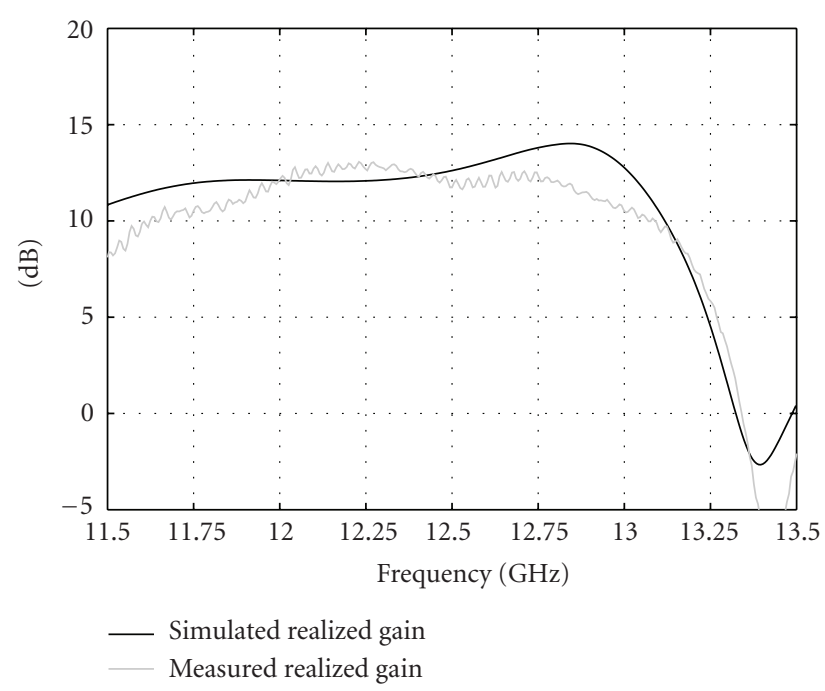

Figure 10: Measured realized gain compared to the simulated one.

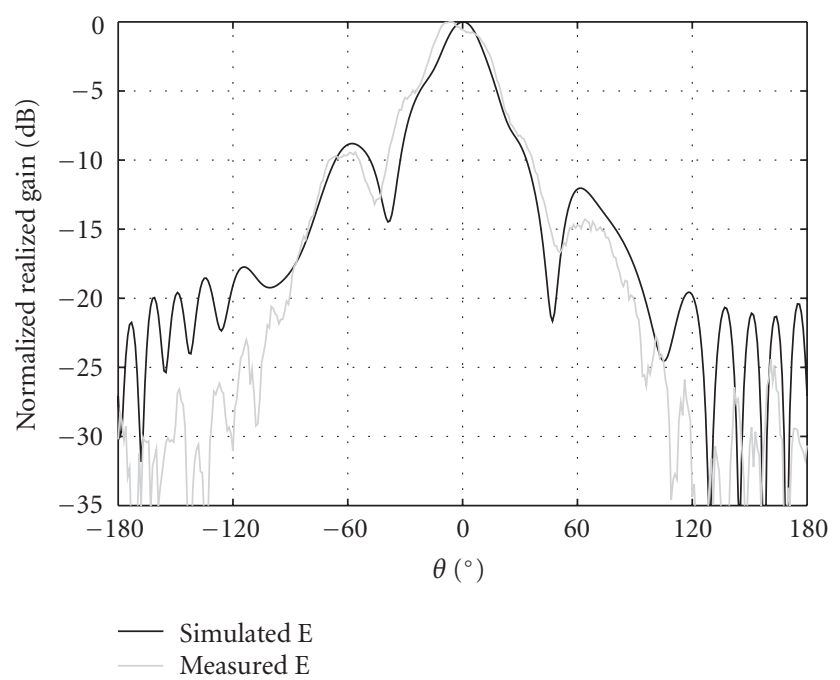

(a)

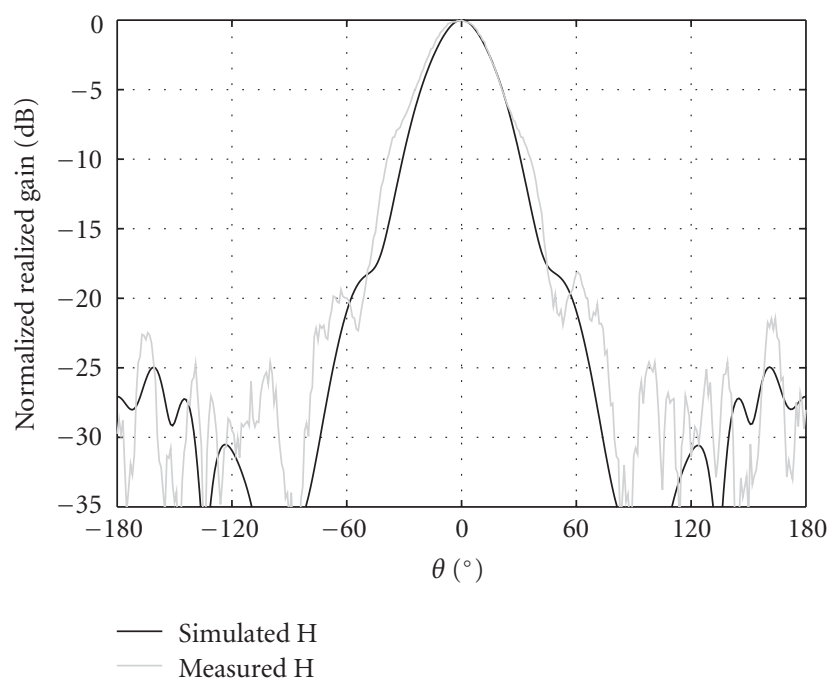

(b)

FIgURE 11: Radiation patterns in the E- and H-plane at $11.9 \mathrm{GHz}$. 


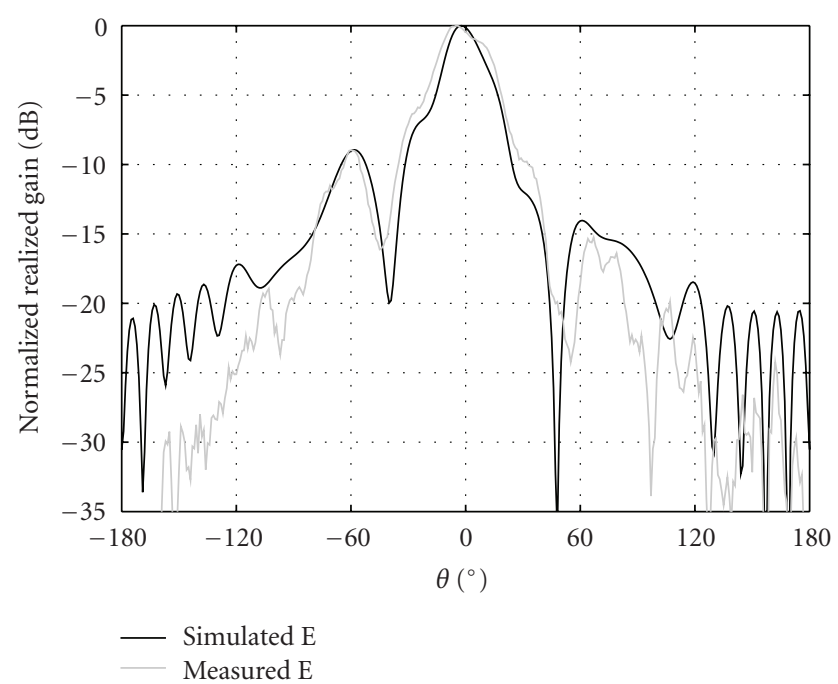

(a)

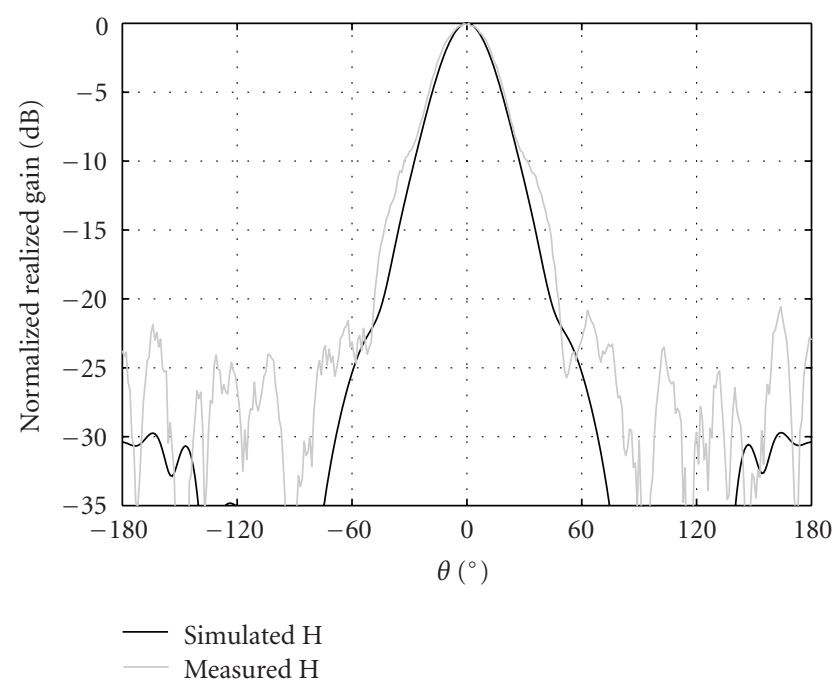

(b)

Figure 12: Radiation patterns in the E- and H-plane at $12.2 \mathrm{GHz}$.

$\left(\left|S_{11}\right|>-10 \mathrm{~dB}\right)$ (Figure 8). The functioning of the antenna is supported by an evanescent mode whose wave impedance is highly imaginary; it is fastidious to transfer the power to this kind of modes. The use of a patch near the ground plane allows exciting this mode, but the patch resonance often excites a narrow frequency band compared to the antenna operation band. This explains the narrow matching band generally obtained with this kind of antennas; a classical EBG antenna is matched $\left(\left|S_{11}\right| \geq-10 \mathrm{~dB}\right)$ in a narrow frequency band around the operating frequency, but the return loss is very high $\left(\left|S_{11}\right|<-3 \mathrm{~dB}\right)$ before the latter. The advantage that can be then taken by the use of the realized antenna is the widening of the matching bandwidth (even though, here it is only equal to $-4 \mathrm{~dB}$ and not $-10 \mathrm{~dB}$ ), so it is approximately equivalent to the radiation bandwidth. The solution which can be proposed to overcome the high $S_{11}$

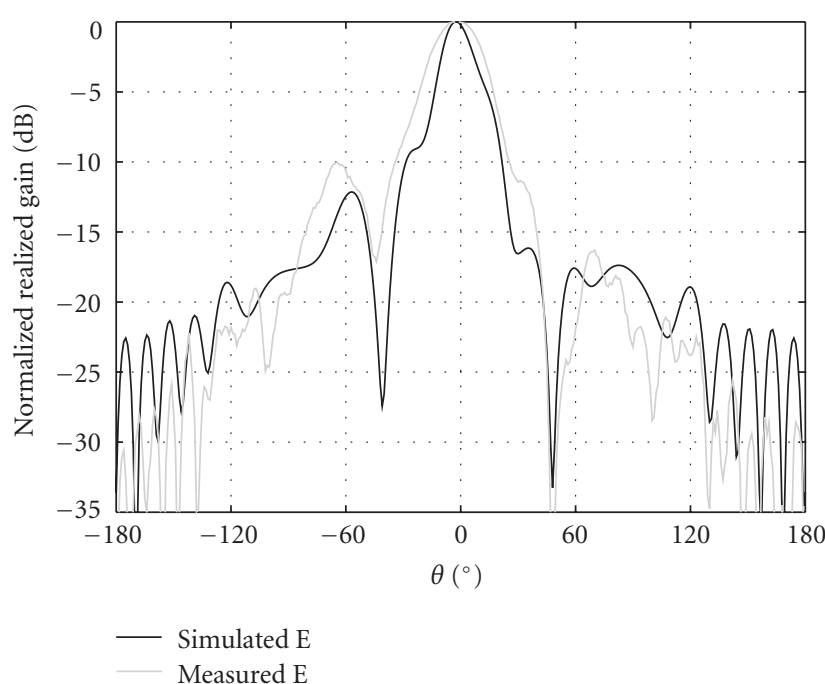

(a)

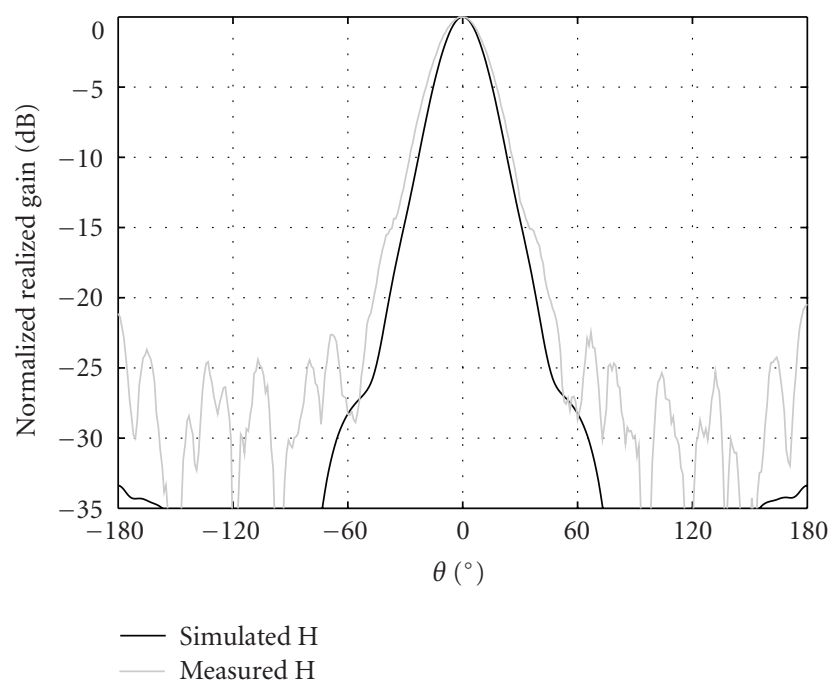

(b)

Figure 13: Radiation patterns in the E- and H-plane at $12.5 \mathrm{GHz}$.

magnitude problem is to approach the multifeed technique. This will be shown in future works. By increasing the feeding sources number, we can improve the matching amplitude given by a simple source. Nevertheless, some precautions should be taken regarding the sources coupling which is modified in presence of the resonator.

3.3. Experimental Validation. The antenna has been realized, its measured return loss is represented in Figure 8 compared to the simulated one, a clear discord between simulated and measured return loss can be noticed. This is due to the patch resonance frequency shift $(500 \mathrm{MHz})$. The shift is proper to the discrepancy of the practical coaxial probe used to feed the patch. The printed patch is represented in Figure 9. The patch resonance shift can be also expressed by the $1.5 \mathrm{~dB}$ of losses in terms of measured realized gain occurring at 


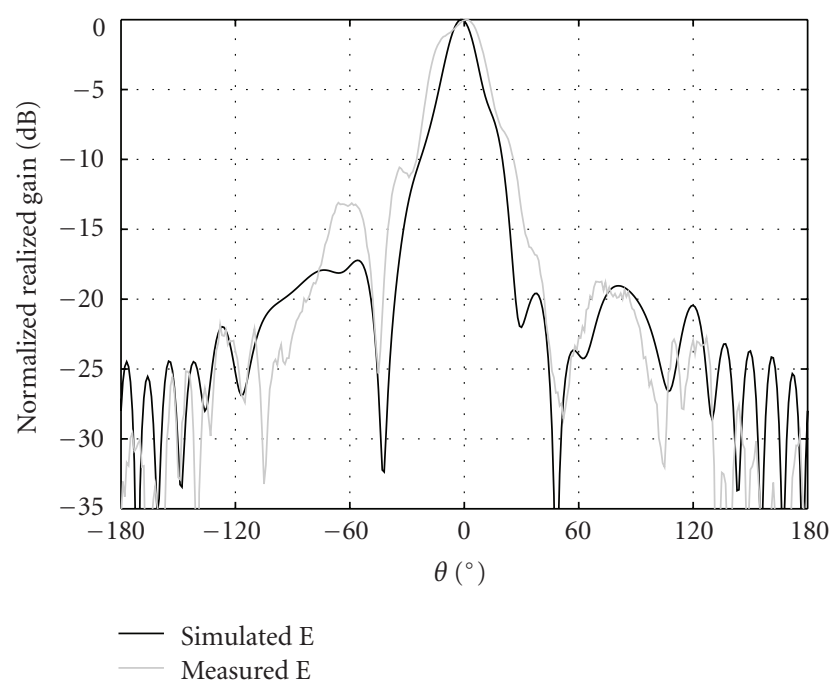

(a)

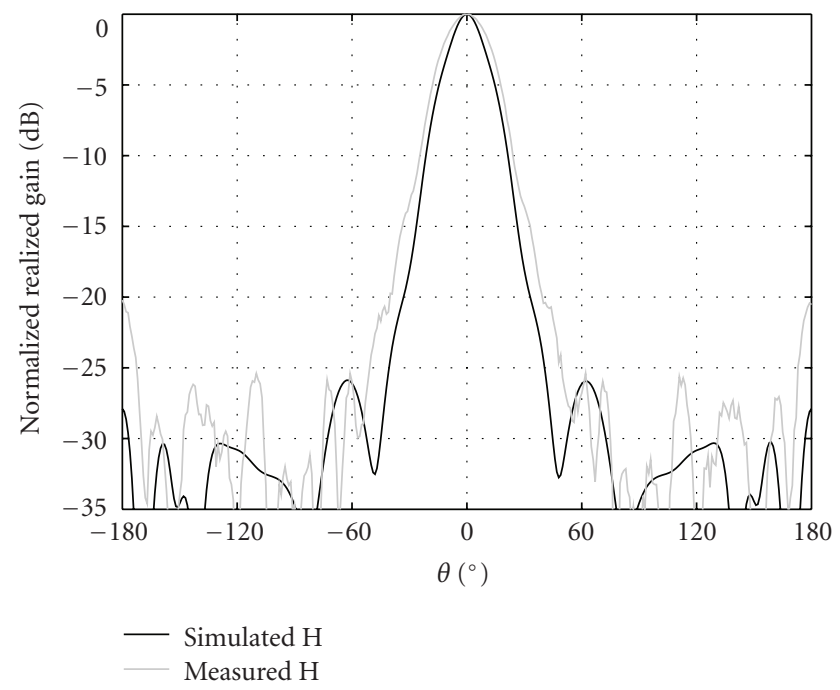

(b)

Figure 14: Radiation patterns in the E- and H-plane at $12.8 \mathrm{GHz}$.

12.75 GHz (Figure 10). However, the radiation bandwidth corresponding to the realized gain is almost kept constant compared to the simulation results.

The measured radiation patterns are shown in the E- and $\mathrm{H}$-planes compared to the simulated ones (Figures 11, 12, 13, 14). A considerably promising agreement between simulations and measurements is noticed. The high side lobes levels in the E-plane and notably at $-60^{\circ}$ can be explained by the presence of the coaxial probe that introduces a dissymmetry in the radiation pattern.

\section{Conclusion}

The phase inversion principle that we have shown in previous works and which results in enlarging the radiation bandwidth of an EBG resonator is taken in here. It is applied to a prototype working in the Ku-band. The EBG resonator antenna is fed by a patch source printed at the proximity of the antenna metallic ground. The prototype is fabricated using the printed circuit technology. The results show a good agreement between the measurement and the simulation.

\section{References}

[1] G. V. Trentini, "Partially reflecting sheet arrays," IRE Transactions on Antennas and Propagation, vol. 4, no. 4, pp. 666-671, 1956.

[2] J. R. James, S. J. Kinany, P. D. Peel, and G. Andrasic, "Leakywave multiple dichroic beamformers," Electronics Letters, vol. 25, no. 18, pp. 1209-1211, 1989.

[3] M. Thevenot, C. Cheype, A. Reineix, and B. Jecko, "Directive photonic-bandgap antennas," IEEE Transactions on Microwave Theory and Techniques, vol. 47, no. 11, pp. 2115-2122, 1999.

[4] A. P. Feresidis and J. C. Vardaxoglou, "High gain planar antenna using optimised partially reflective surfaces," IEE Proceedings: Microwaves, Antennas and Propagation, vol. 148, no. 6, pp. 345-350, 2001.

[5] R. Gardelli, M. Albani, and F. Capolino, "Array thinning by using antennas in a Fabry-Perot cavity for gain enhancement," IEEE Transactions on Antennas and Propagation, vol. 54, no. 7, pp. 1979-1990, 2006.

[6] E. Yablonovitch, "Photonic band-gap structures," Journal of the Optical Society of America B, vol. 10, no. 2, pp. 283-295, 1993.

[7] C. Cheype, C. Serier, M. Thevenot, T. Monedière, A. Reineix, and B. Jecko, "An electromagnetic band gap resonator antenna," IEEE Transactions on Antennas and Propagation, vol. 50, no. 9, pp. 1285-1290, 2002.

[8] L. Leger, C. Serier, R. Chantalat, M. Thevenot, T. Monedière, and B. Jecko, "1D dielectric electromagnetic band gap (EBG) resonator antenna design," Annales des Télécommunications, vol. 59, no. 3-4, pp. 242-260, 2004.

[9] D. R. Jackson and N. G. Alexopoulos, "Gain enhancement methods for printed circuit antennas," IEEE Transactions on Antennas and Propagation, vol. 33, no. 9, pp. 976-987, 1985.

[10] S. Wang, A. P. Feresidis, G. Goussetis, and J. C. Vardaxoglou, "Low profile highly directive antennas using EBG superstrates and metamaterial ground planes," in IEEE Antennas and Propagation Society International Symposium, vol. 4B, pp. 335338, Washington, DC, USA, July 2005.

[11] A. R. Weily, K. P. Esselle, T. S. Bird, and B. C. Sanders, "Linear array of woodpile EBG sectoral horn antennas," IEEE Transactions on Antennas and Propagation, vol. 54, no. 8, pp. 2263-2274, 2006.

[12] M. Hajj, E. Rodes, D. Serhal, et al., "Metallic EBG sectoral antenna for base stations," in Proceedings of the 19th International Conference on Applied Electromagnetics and Communications (ICECom '07), pp. 1-4, Dubrovnik, Croatia, September 2007.

[13] G. K. Palikaras, A. P. Feresidis, and J. C. Vardaxoglou, "Cylindrical electromagnetic bandgap structures for directive base station antennas," IEEE Antennas and Wireless Propagation Letters, vol. 3, no. 1, pp. 87-89, 2004.

[14] H. Chreim, E. Pointereau, B. Jecko, and P. Dufrane, "Omnidirectional electromagnetic band gap antenna for base station applications," IEEE Antennas and Wireless Propagation Letters, vol. 6, pp. 499-502, 2007. 
[15] L. Moustafa and B. Jecko, "Bandwidth improvement of EBG resonator antennas using double-layer FSS," International Journal of Antennas and Propagation, vol. 2008, Article ID 315052, 5 pages, 2008.

[16] L. Moustafa, M. Thevenot, T. Monediere, and B. Jecko, "Design method of EBG material with wide defect band," in Proceedings of the European Conference on Antennas and Propagation (EuCAP '09), pp. 3235-3239, 2009.

[17] J. Drouet, Electromagnetic analytical methods dedicated to the design of multi source EBG resonator antennas, Ph.D. thesis, University of Limoges, Limoges, France, 2007.

[18] L. Leger, T. Monediere, and B. Jecko, "Enhancement of gain and radiation bandwidth for a planar 1-D EBG antenna," IEEE Microwave and Wireless Components Letters, vol. 15, no. 9, pp. 573-575, 2005. 

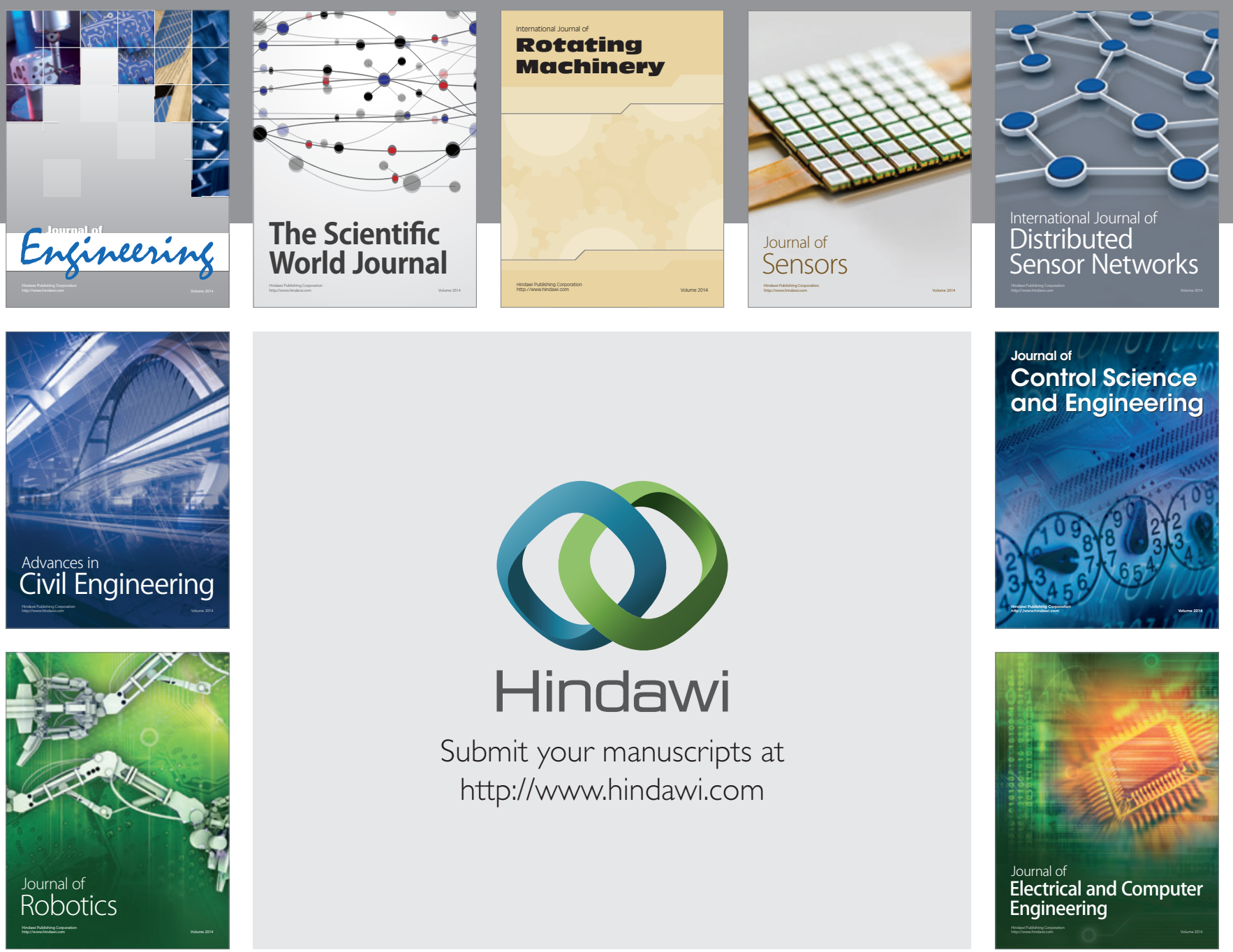

Submit your manuscripts at

http://www.hindawi.com
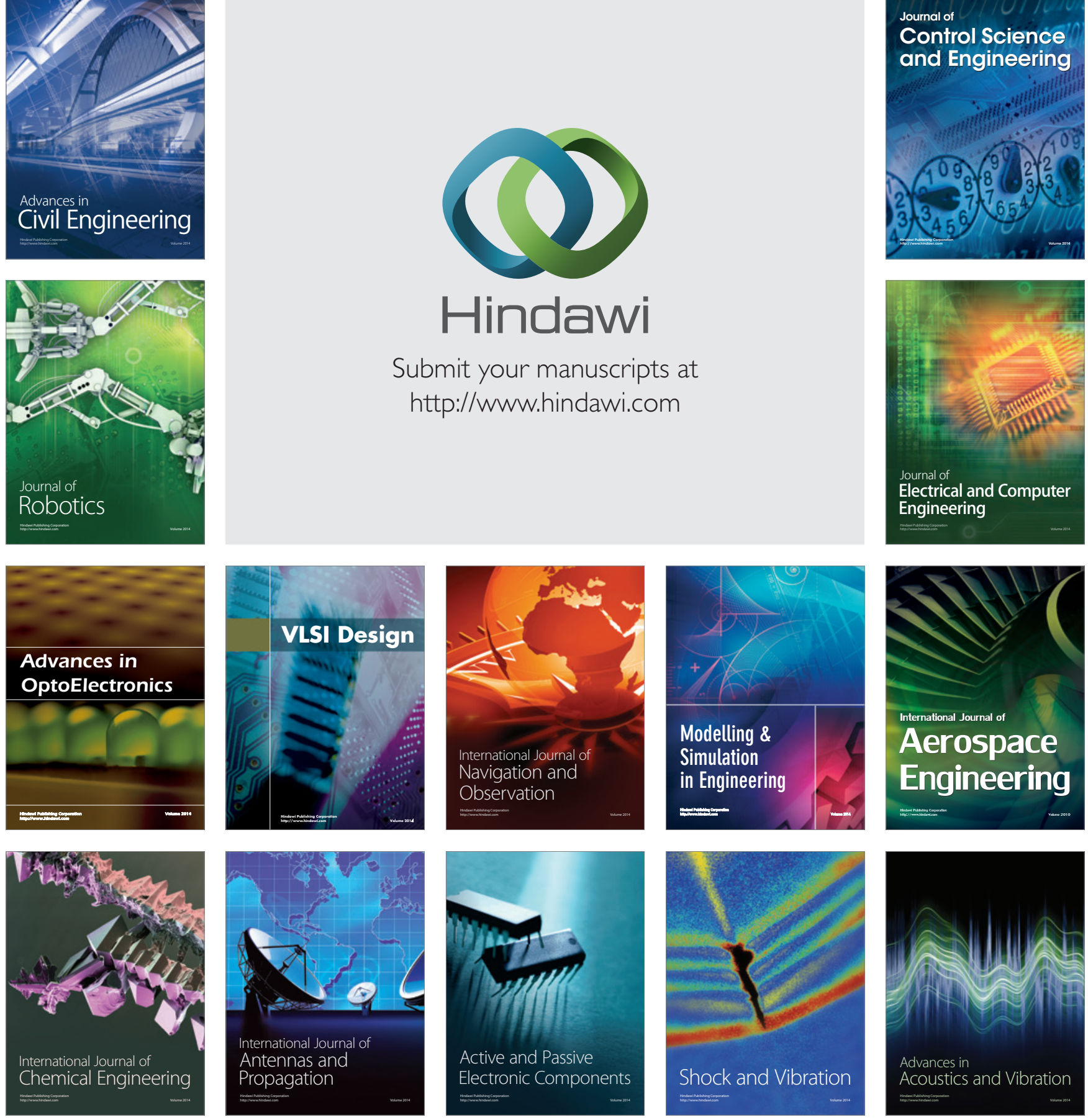